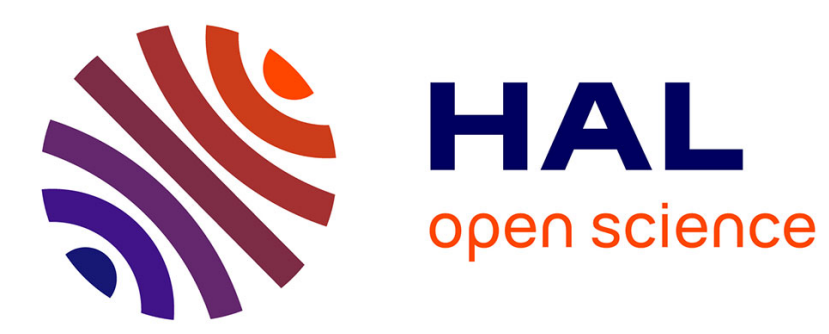

\title{
Boundary Condition in Liquid Thin Films Revealed through the Thermal Fluctuations of Their Free Surfaces
}

B Pottier, Christian Frétigny, L Talini

\section{To cite this version:}

B Pottier, Christian Frétigny, L Talini. Boundary Condition in Liquid Thin Films Revealed through the Thermal Fluctuations of Their Free Surfaces. Physical Review Letters, 2015, 114 (22), pp.227801. 10.1103/PhysRevLett.114.227801 . hal-01176182

\section{HAL Id: hal-01176182 \\ https://hal.sorbonne-universite.fr/hal-01176182}

Submitted on 15 Jul 2015

HAL is a multi-disciplinary open access archive for the deposit and dissemination of scientific research documents, whether they are published or not. The documents may come from teaching and research institutions in France or abroad, or from public or private research centers.
L'archive ouverte pluridisciplinaire HAL, est destinée au dépôt et à la diffusion de documents scientifiques de niveau recherche, publiés ou non, émanant des établissements d'enseignement et de recherche français ou étrangers, des laboratoires publics ou privés. 


\title{
Boundary condition in thin liquid films revealed through the thermal fluctuations of their free surfaces
}

\author{
B. Pottier ${ }^{1,2}$, C. Frétigny ${ }^{1,2}$, L. Talini ${ }^{1,2}$ \\ ${ }^{1}$ École Supérieure de Physique et de Chimie Industrielles de la Ville de Paris (ESPCI) \\ ParisTech, PSL Research University, Sciences et Ingénierie de la matière Molle, CNRS \\ UMR7615, 10, Rue Vauquelin, F-75231 Paris Cedex 05. France. \\ ${ }^{2}$ Sorbonne-Universités, UPMC Univ. Paris 06, SIMM, 10, Rue Vauquelin, F-75231 Paris \\ Cedex 05, France.
}

\begin{abstract}
We investigate the properties of nanometric liquid films with a new non invasive technique. We measure the spontaneous thermal fluctuations of the free surfaces of liquids to probe their hydrodynamic boundary condition at a solid wall. The surface fluctuations of a silicon oil film could be described with a no-slip boundary condition for film thicknesses down to $20 \mathrm{~nm}$. Oppositely, a $4 \mathrm{~nm}$ negative slip length had to be introduced to describe the behavior of n-hexadecane, consistently with previous Surface Force Apparatus data on the same system. Our results demonstrate that at vanishing flow a nanometric solid-like layer close to the wall may exist according to the nature of the liquid.
\end{abstract}

Significant modifications of the properties of liquids at nanometric distances from a solid wall have been evidenced in the past decades [1], but a unifying picture of the different reported features (see for instance [2-7]) is still lacking. The questions at stake are in particular crucial for transport properties at small scales, involved e.g. in flow through nanoporous materials or in nanofluidic devices [1]. In the past years, the investigation of the behavior of nanometric fluids has benefited from experimental developments enabling the measurement of both distances down to $0.1 \mathrm{~nm}$ and forces down to $1 \mathrm{pN}$, in particular with Surface Force Apparatus (SFA) [8] or Atomic Force Microscopy (AFM, see for example [7, 9-11]). Alternatively, techniques based on fluorescence microscopy [4, 12-16] or X-ray scattering $[17,18]$ have been employed. In most experiments, a flow is induced in the confined liquid and the measurement of either pressure or displacement is compared to the behavior at large scales. Different deviations from macroscopic hydrodynamics have been observed, among which a boundary condition at the solid surface differing from the classical zero velocity [19]: some liquids were observed to slip at the solid surface, or oppositely to behave as an immobile layer 
in its vicinity, sometimes attributed to an ordering of the liquid molecules. In terms of the classically defined slip length $b$, i.e. the length at which the extrapolated fluid velocity is zero, slip corresponds to $b>0$, whereas an immobile layer is described with $b<0$. In the past years, the contradictory nature of some experimental results on the very same systems has been attributed to the crucial role of surface roughness [19]. More recently, it was demonstrated that, when a nanometer-thick fluid layer is confined between solid walls, the elastic deformation of the solid surfaces coupled with the fluid flow may dominate the mechanical response [21]. In addition, AFM measurements may depend on the cantilever geometry [10]. Measurements at those scales are indeed challenging, and less invasive techniques are needed to probe the flow properties of nanometric fluids.

Along that line, experiments taking profit of thermal fluctuations to characterize flow properties have been recently developed [12, 22]. External forcing is then replaced by spontaneous motion resulting from thermal energy. For instance, measurements of the diffusion of Brownian tracers were used to characterize boundary conditions in liquids [12]. The obtained results were in agreement with theoretical predictions [20] and have been more recently confirmed with other techniques [10,11,13-16]: slip is expected with non wetting fluids, and slip lengths are of the order of tens of nanometers. However, the immobile fluid layers observed in the 80 's by several authors for wetting liquids [2, 3] remain to be confirmed. Such layers are expected to be only a few nanometers thick, and an even better resolution than in ref. [12] is needed.

In the present Letter, we measure the spontaneous thermal fluctuations of the free surfaces of thin liquid films in order to probe the hydrodynamic boundary condition at the solid/liquid interface. The film thinning is thermally driven and its thickness is measured by interferometry. We show that the fluctuations of a silicon oil film can be described with the classical no slip boundary condition at the solid surface for thicknesses down to $20 \mathrm{~nm}$. Oppositely, for a n-hexadecane film on a glass substrate, the dynamics of the surface fluctuations is slowed down, revealing the presence of a few nanometer thick immobile layer close to the solid surface, in agreement with previous findings [2, 3].

The liquid films are prepared as follows: the oils are filtered and further spin coated on a smooth colored glass substrate (Hoya Longpass optical filter). The viscosity $\eta$ and surface tension $\gamma$ are respectively $\eta=18 m P a . . s$ and $\gamma=20.5 \mathrm{mN} . \mathrm{m}^{-1}$ for silicon oil (Rhodorsil v20) and for n-hexadecane $\eta=2.7 m P a . . s$ and $\gamma=27.5 \mathrm{mN} . \mathrm{m}^{-1}$. The initial thickness of the oil 
films is smaller than $1 \mu \mathrm{m}$, and it is further decreased using a thermocapillary effect [23, 24], with a more accurate control than spontaneous spreading.

As described in the following, the experimental set up uses a single laser beam (Fig. 1), which serves to simultaneously i) thin down the liquid film, ii) measure its thickness and iii) measure the fluctuations of its free surface. The laser $(\mathrm{He} / \mathrm{Ne}$, wavelength $\lambda=632.8 \mathrm{~nm}$, nominal power $30 \mathrm{~mW}$ ) is focused with a microscope objective (Olympus x20 or x50) at the surface of the liquid film. The radius of the beam at the surface is either $R=3.50 \mu \mathrm{m}$ or $R=1.54 \mu \mathrm{m}$.

The glass substrate on which the liquid film lies is heated by the laser, which results in a rise of the temperature of the liquid through thermal diffusion. The radial temperature gradient induces a surface tension gradient, resulting in a Marangoni stress $\vec{\nabla} \gamma=(d \gamma / d T) \vec{\nabla} T$ exerted on the liquid surface [23], where $d \gamma / d T \approx-10^{-4} N \cdot m^{-1} \cdot K^{-1}$. As schematized in Fig. 1 , the liquid flows away from the heated region, and the liquid film thins down locally. The temperature rise of the liquid was measured with an infra-red camera to be smaller than $1 \mathrm{~K}$ and, owing to the weak absorption by the substrate and strong focalization of the laser beam, the horizontal size of the heated region is of several spot radii. Within those conditions, the interface remains flat at the scale of the laser beam, and the resulting flow in the liquid film has no significant effect on the surface fluctuations, as shown in the following. In addition, no effect of the laser heating on the fluid viscosity or refractive index could be measured.

The same laser allows for precise measurement of the film thickness by interferometry; the refractive index $n_{s}=1.555$ of the glass substrate differing from the oil indexes (respectively $n=1.400$ and $n=1.434$ for silicon oil and hexadecane) the transmitted part of the beam reflects off the liquid/solid interface and further interferes with the reflection at the air/liquid interface. In practice, the interfering beam is centered on two quadrants of a photodiode and, after current to voltage conversion, $S_{0}$, the sum of the voltages delivered by the two quadrants is measured as a function of time. As the film thickness $h$ decreases with time, $S_{0}(t)$ varies sinusoidally with a phase given by $4 \pi \mathrm{nh} / \lambda$, and the thickness variations can be fully determined [25]. 


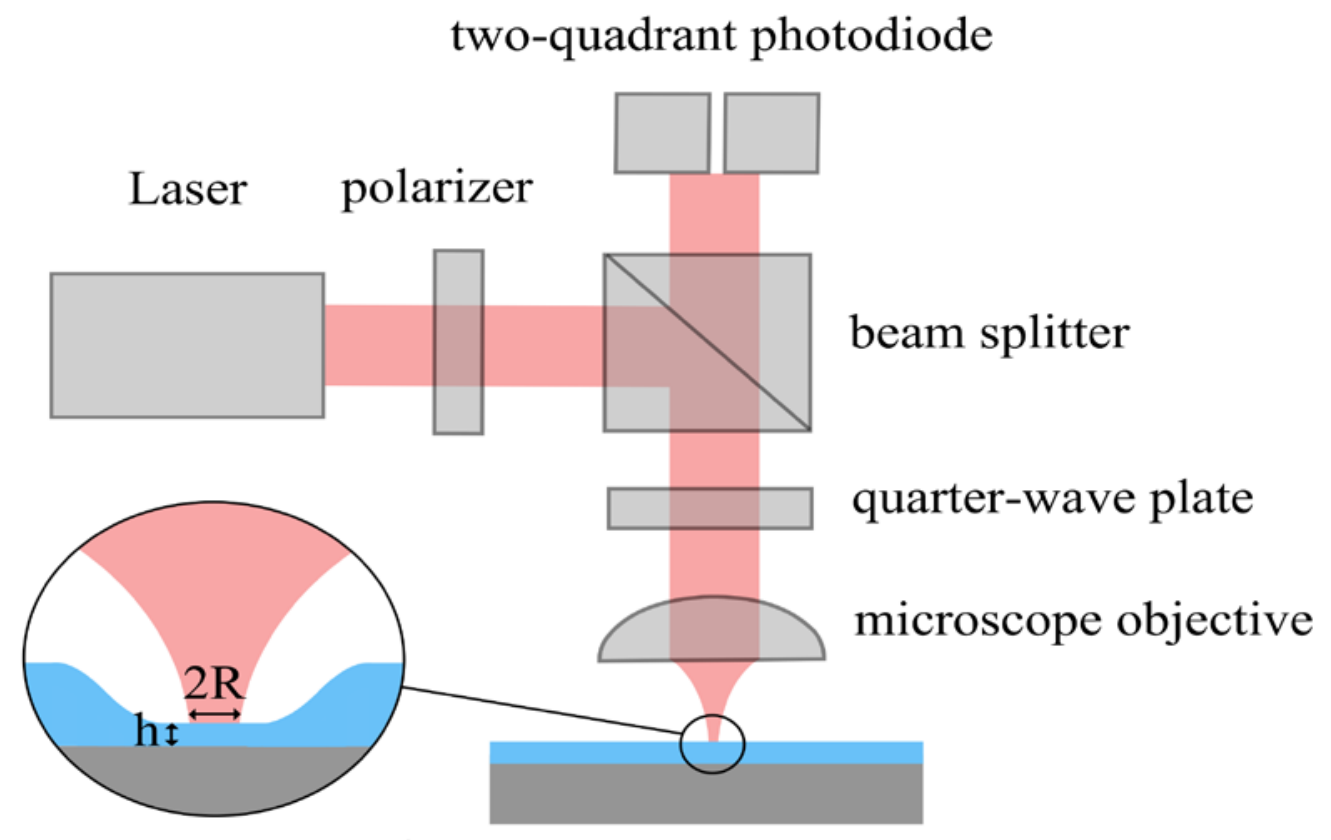

FIG. 1: (Color online) Schematized experimental set-up. The laser is focused with a microscope objective at the surface of a liquid film lying on a glass substrate. Its reflection is centered on the two quadrants of the photodiode. The laser serves to i) thin down the film thanks to a thermocapillary effect, while its surface remains flat at the laser beam radius scale (detailed view, not to scale) ii) measure the film thickness by analyzing the total light intensity on the two quadrants, which results from the interferences of the air/liquid and liquid/solid reflections, iii) measure the surface thermal fluctuations of the film by analyzing the fluctuations in the difference of light intensity on the two quadrants. The polarizer and quarter-wave plate suppress any back reflection of the laser.

Finally, in addition to thickness actuation and monitoring, the laser beam is used to probe the thermal fluctuations of the free surface of the liquid films: the beam that reflects at the air/film interface is deflected by the corrugation of that interface, and the fluctuations of its position can be related to the ones of the interface height, as previously shown [26]. More precisely, as the liquid film thins down, we digitize the preamplified difference of the voltages delivered by the two quadrants of the photodiode on which the beam is centered. The duration of an acquisition is small compared to the characteristic time of thickness decrease, and the 
sampling rate is $200 \mathrm{kHz}$. For each acquisition, the power spectral density (PSD) denoted as $S(\omega)$, is computed and the PSD's are further recorded at a rate of 38 per minute.

Since the beam is specularly reflected by the surface, $S(\omega)$ measures the contribution of all the spatial modes of the fluctuating surface. However, the main contribution comes from the modes of wavelength close to the beam size at the surface, $R$, which sets the measurement scale. In the experiments described herein, the amplitude of $S(\omega)$ depends on both the laser intensity and the film thickness, owing to the interferences between the reflections at the air/liquid and liquid/solid interfaces. For a film of thickness $h$, optical index $n$, and laser beam of wavelength $\lambda, S(\omega)$ writes [26]:

$$
S(\omega)=K^{2}\left[A+B \cos \left(\frac{4 \pi n h}{\lambda}\right)\right]_{0}^{2+\infty} P(\omega, k) \Phi(k) k d k
$$

The prefactor results from the thickness dependence of the intensity of the interfering laser beam. The term within the integral corresponds to the contribution of each spatial mode $k$ of the surface with $P(\omega, k)$ being the PSD of the height of the free surface and $\Phi(k)$ a weighting function that only depends on the wavelength and divergence of the laser beam, and is maximum for $k \approx 1 / R$ [17]. For a medium in thermal equilibrium, $P(\omega, k)$ is related to the imaginary part of the liquid mechanical susceptibility through a fluctuation dissipation relation [27].

In the absence of confinement, i.e. for thickness $h>>R, P(\omega, k)$ only depends on the liquid properties, namely viscosity $\eta$ and surface tension $\gamma$ for simple liquids in an overdamped regime [28]. When the film thickness $h$ is smaller than the beam radius $R$, as in the experiments reported herein, the effect of confinement has to be taken into account [29-31]. The function $P(\omega, k)$ becomes dependent on both the thickness $h$ and the boundary condition at the liquid/solid interface, which can be described by introducing a slip length $b$ and written under the form [30]:

$$
P(\omega, k)=\frac{k_{B} T}{\pi k^{2} \tilde{\omega}(k)} \frac{1}{1+\left(\frac{\omega}{\tilde{\omega}(k)}\right)^{2}}
$$

Where $k_{B} T$ is the thermal energy. The characteristic frequency $\tilde{\omega}(k)$ depends on the liquid properties as well as on $h$ and $b$ [30]. For film thicknesses below a few tens of nanometers, Van der Waals interactions are expected to modify the dynamics of surface fluctuations [17]. 
However, for the film thicknesses probed herein, those effects are limited to frequencies smaller than $1 \mathrm{~Hz}$ [26], whereas we probe frequencies larger than $100 \mathrm{~Hz}$.

Therefore, concomitant optical measurements of the thickness and of the surface fluctuations of a liquid film can be conducted. For a liquid of known viscosity and surface tension, the boundary condition at the solid substrate - characterized by a slip length $b$ - can be inferred from the fluctuation spectrum with a precision that depends on the film thickness. We first focus on measurements conducted with relatively thick films. In Fig. 2, we show PSDs recorded during the spreading of a silicon oil film, that correspond to intensity maxima of the interfering beams (i.e. film thicknesses multiples of $\lambda / 2 n=226 n m$ ), and thus to a given constant prefactor in Eq. (1).

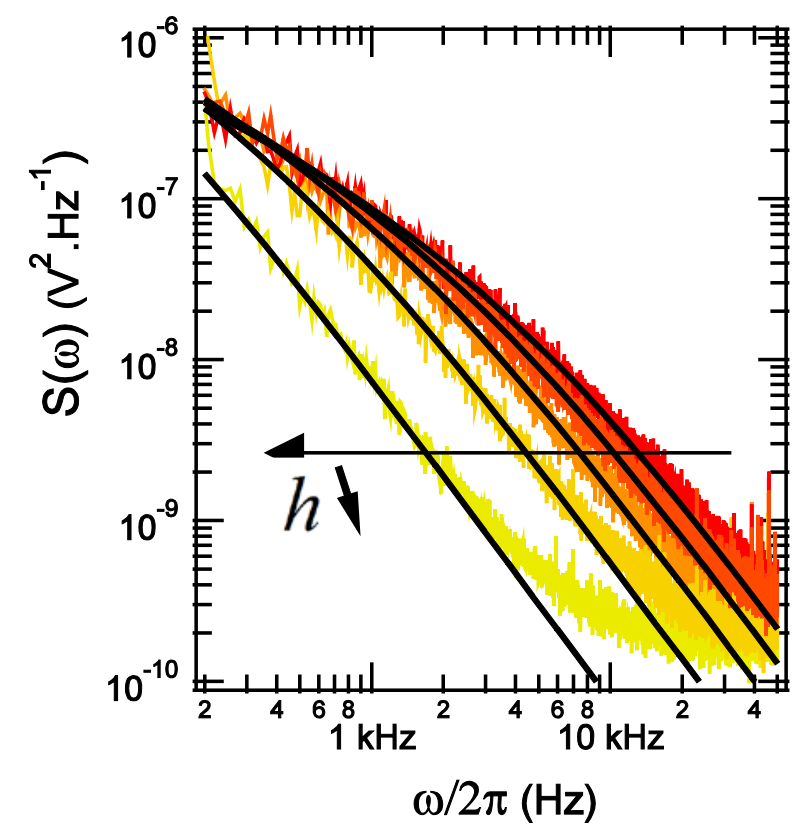

FIG . 2: (Color online) Surface fluctuation spectra of a silicon oil film. The spectra were recorded at different instants of the film spreading, corresponding to thicknesses determined by interferometry and equal to multiples of $\lambda / 2 n=226 n m$, from $5 \lambda / 2 n$ to $\lambda / 2 n$ from right to left. The predicted spectra (black lines) were computed for each thickness using Eq. (1) and (2), the silicon oil properties and $R=3.50 \mu \mathrm{m}$. The poor description of the spectra at high frequency results from the set-up noise.

As observed in Fig. 2, for increasing confinement, the fluctuation dynamics shifts to lower frequencies, and both the shapes and amplitudes of the $S(\omega)$ curves evolve. Such evolutions can be understood by examining the asymptotic limits of $S(\omega)$. According to Eq. (2), for large frequencies $\omega>>\widetilde{\omega}, P(\omega, k)$ is expected to vary as $\tilde{\omega}(k) /(\omega k)^{2}$. For strong 
confinement, i.e. $k h<<1$, and slip length such that $b k<<1$, the characteristic frequency $\tilde{\omega}$ can be developed as $\tilde{\omega} \approx k^{4} h^{3}(1+3 b / h) / 3 \eta$ [30]. Finally, a simple estimation of $S(\omega)$ at high frequency and small thickness is found by writing $k \approx 1 / R$ and by neglecting the dependency on $h$ of the prefactor, (i.e. assuming $h<<\lambda$ ) in Eq. (1), which yields:

$$
S(\omega) \propto \frac{k_{B} T}{3 \pi \eta \lambda^{2} R^{4} \omega^{2}} h^{3}\left(1+\frac{3 b}{h}\right)
$$

Therefore, as confinement increases, $\tilde{\omega}$ decreases, and the high frequency scaling $S(\omega) \propto \omega^{-2}$ is shifted to lower frequencies together with a strong decrease of the amplitude of $S(\omega)$. The data of Fig. 2 are not only in qualitative agreement with those trends, but are also fully described by the numerically computed PSD (full black lines) following the complete expression given by Eq. (1) together with Eq. (2) and a slip length $b=0$. The same quantitative agreement was obtained for experiments performed at different laser powers, thus confirming that the physical properties of the liquids are not significantly modified by laser heating in the investigated range. In addition, although a flow is induced in the liquid by the thermocapillary stress, the surface fluctuations are well described by considering the liquid is at rest, although the resulting shear is expected to modify their dynamics [33]. However, in the present experiment, the shear rate can be estimated as $\dot{h} R / h^{2}$ and remains smaller than $10 s^{-1}$; the characteristic time associated with the height fluctuations of the surface being smaller than $1 \mathrm{~ns}$, the associated Peclet number is smaller than $10^{-8}$, and the effect of shear completely negligible [33].

In summary, the predictions for the surface fluctuations of a liquid in equilibrium fully agree with the measurements of Fig. 2. A no slip condition at the solid surface lead to a correct description of the data, but in those measurements performed on relatively thick films, the accuracy on the slip length is very poor. Actually, slip length for wetting liquids are expected to be only of a few molecular sizes and, according to Eq. (3), film thicknesses of the same order of magnitude as the slip length must be reached in order to measure a significant effect of slip on surface fluctuations.

In order to accurately determine slip lengths, experiments were performed with stronger beam focalization with beam radius $R=1.54 \mu \mathrm{m}$. The laser power was such that the high-frequency noise limit was small enough to measure the surface fluctuations of films with thicknesses down to $20 \mathrm{~nm}$. For different frequencies $\omega$, the quantities $\omega^{2} S(\omega)$ were averaged over a logarithmic range from each recorded fluctuation spectrum. The variations of $\omega^{2} S(\omega)$ are 
reported as a function of the film thickness for silicon oil (Fig. 3) for two frequencies $\omega$, together with the theoretical expressions (dotted lines). In agreement with the scaling of Eq. (3), $\omega^{2} S(\omega)$ follows a master curve for small thicknesses whatever the frequency. In that limit, the fluctuation measurement is sensitive to the value of the slip length $b$, and the data is well described with $b=0 \pm 0.5 \mathrm{~nm}$ for silicon oil, consistently with measurements on wetting fluids [12]. The accuracy of the slip length $b$ is deduced from the quality of the master curve. Uncertainties on the different parameters such as the liquid viscosity or laser beam radius do not significantly affect the value of $b$, but only of the prefactor in Eq. (3), which is accurately determined using data at larger thicknesses.

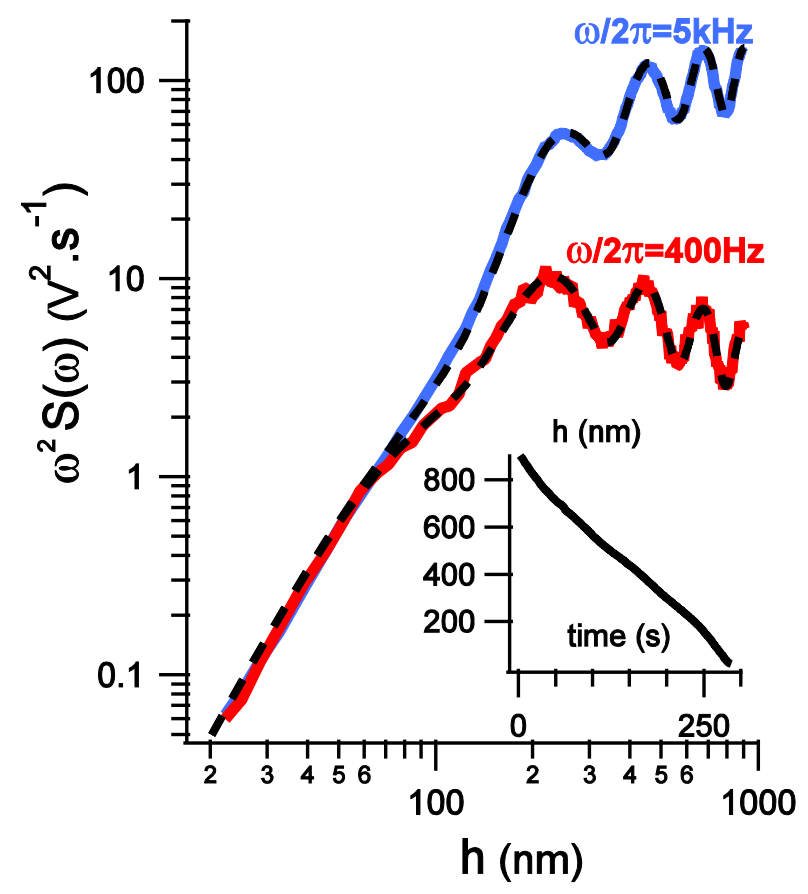

FIG. 3: (Color online) Surface fluctuation data of a silicon oil film, whose thickness $h$ decreases with time (inset). The quantity $\omega^{2} S(\omega)$ shown as a function of film thickness was computed from the fluctuation spectra $S(\omega)$, and corresponds to an average over a logarithmic range centered on frequency $\omega$. For each frequency, the predicted value of $\omega^{2} S(\omega)$ was computed with Eq. (1) and (2), with the same parameters as in Fig.2 but a laser beam radius $R=1.54 \mu \mathrm{m}$ and the amplitude and the slip length $b=0$, which were adjusted to fit to the data.

Similar experiments conducted with a n-hexadecane film (Fig. 4) show significantly slower fluctuation dynamics at small thicknesses. As a result, a negative slip length $b=-4 \pm 0.5 \mathrm{~nm}$ is used to fully describe the variations of $\omega^{2} S(\omega)$, that value being the same at all frequencies 
(black full lines in Fig. 4). Negative slip lengths with similar values have previously been reported in experiments with hexadecane [2, 3]. They have been attributed to the presence close to the solid wall of a layer of properties differing from the bulk, which can conveniently be described as an apparent immobile layer. The thickness of that layer is of several molecular sizes, in agreement with experimental but also numerical studies, which reported boundary conditions depending strongly on the nature of the fluid and its interactions with the solid wall [34, 35]. In particular, layering in chain molecule fluids such as n-hexadecane is expected to be more important than in other fluids, e.g. in branched molecule fluids.

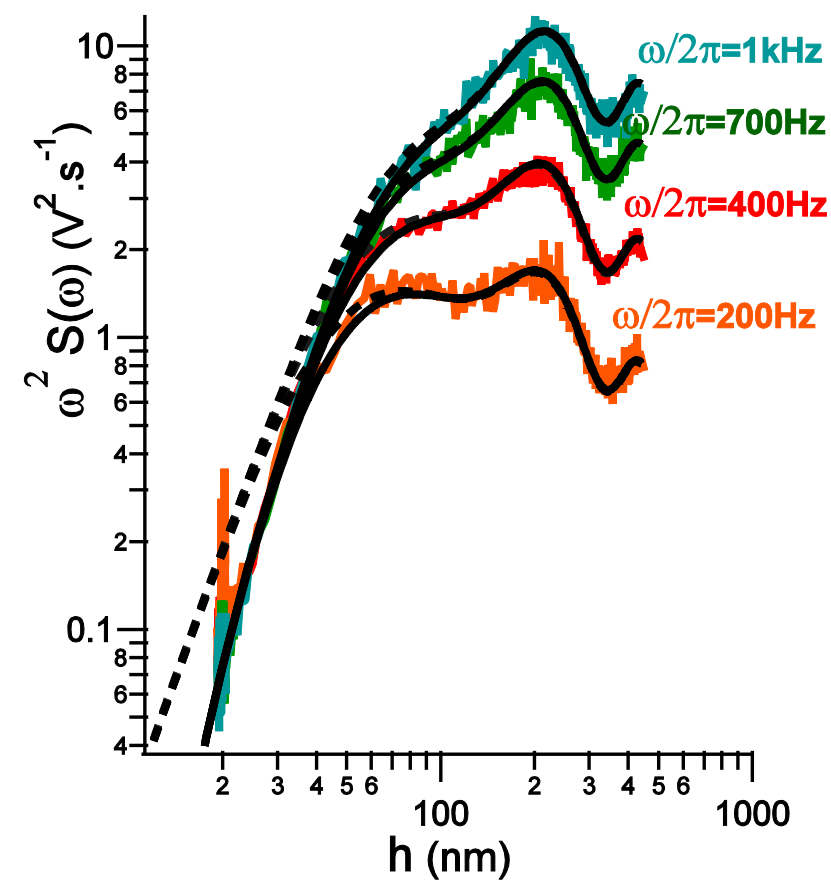

FIG. 4: (Color online) Same as Fig. 3 for a n-hexadecane film on a glass substrate. At small thicknesses, the data deviates from the zero slip prediction (dotted line), but is fully described with a negative slip length $b=-4 n m$ (full black line), for all probed frequencies.

Our results are complementary to previous SFA experiments [2,3] since they demonstrate that neither shear nor confinement between two solid surfaces is needed for the formation of the immobile layer. They are in apparent contradiction with other experiments in which positive slip lengths of the order of $100 \mathrm{~nm}$ were measured when hexadecane was made to flow on a glass substrate [4]. However, the shear rate exerted in the latter experiments was very large (from $10^{2} \mathrm{~s}^{-1}$ to $10^{4} \mathrm{~s}^{-1}$ ), and it is plausible that the solid-like layer is substantially modified at those shear rates, or even is displaced as a whole, which would result in a large slip length. 
Finally, the existence of a layer with different properties close to the solid wall is similar in nature to results reported in terms of solvation forces measured in compressed thin films [5, 6]. Drastic changes in mechanical properties, such as viscoelastic behavior with relaxation times very large compared to molecular ones, were reported in such films [6, 9]. Interestingly, the behavior we observe for frequencies varying over a decade can be described with the same slip length, showing no frequency dependence in that range. Further investigation is however needed to understand the nature of the so called immobile layer.

In conclusion, using the measurement of free surface fluctuations of thin liquid films, we have probed the hydrodynamic boundary condition at a solid surface in two wetting fluids for vanishing flow. We have evidenced the presence of an immobile layer close the wall that is dependent on the nature of the liquid. Our results open new perspectives for the investigation of the properties of nanometric liquid films.

Acknowledgements: we thank T. Bickel and E. Charlaix for fruitful discussions, and L. Michel for the measurements with the infra-red camera. 


\section{REFERENCES}

[1] L. Bocquet, and E. Charlaix, Chem. Soc. Rev. 39, 1073 (2010).

[2] D. Y. C. Chan, and R. G. Horn, J. Chem. Phys. 83, 5311 (1985).

[3] J. M. Georges et al., J. Chem. Phys. 98, 7345 (1993).

[4] R. Pit, H. Hervet, and L. Leger, Phys. Rev. Lett. 85, 980 (2000).

[5] J. Klein, and E. Kumacheva, Science 269, 816 (1995).

[6] A. L. Demirel, and S. Granick, Phys. Rev. Lett. 77, 2261 (1996).

[7] A. Maali, T. Cohen-Bouhacina, G. Couturier and J.P. Aimé, Phys. Rev. Lett. 96, 086105 (2006).

[8] J. Israelachvili, Intermolecular and Surface Forces (Academic, London, 1992).

[9] T.-D. Li, and E. Riedo, Phys. Rev. Lett. 100, 106102(2008).

[10] C. L. Henry, and V. S. J. Craig, Physical Chemistry Chemical Physics 11, 9514 (2009).

[11] L. Zhu, P. Attard and C. Neto, Langmuir 28, 7768 (2012).

[12] L. Joly, C. Ybert, and L. Bocquet, Phys. Rev. Lett. 96, 046101 (2006).

[13] D. Lasne et al., Phys. Rev. Lett. 100, 214502 (2008).

[14] O. I. Vinogradova et al., Phys. Rev. Lett. 102, 118302 (2009).

[15] D. Schäffel et al., Phys. Rev. E 87, 051001 (2013).

[16] Z. Li et al., J. Fluid Mech. 766, 147 (2015)

[17] I. M. Tidswell et al., Phys. Rev. Lett. 66, 2108 (1991).

[18] C. Gutt et al., Phys. Rev. Lett. 99, 096104 (2007).

[19] E. Lauga, M. Brenner, and H. Stone in Springer handbook of experimental fluid mechanics, 1219-1240, Springer, New-York, 2007.

[20] L. Bocquet, and J. L. Barrat, Soft Matter 3, 685 (2007).

[21] R. Villey et al., Phys. Rev. Lett. 111, 215701 (2013).

[22] F. Liu et al., Phys. Rev. E 87, 062406 (2013).

[23] J. P. Burelbach, S. G. Bankoff, and S. H. Davis, Physics of Fluids a-Fluid Dynamics 2, $322(1990)$.

[24] H. M. J. M. Wedershoven et al., Appl. Phys. Lett. 104, 054101 (2014).

[25] See Supplemental Material at http://links.aps.org/supplemental/

[26] A. Tay et al., Rev. Sci. Instrum. 79, 7 (2008).

[27] M. A. Bouchiat and J. Meunier, Journal De Physique 32, 561-571 (1971).

[28] D. Langevin, Light Scattering by Liquid Surfaces and Complementary Techniques, Marcel Dekker, New-York, 1992. 
[29] J. Jackle, J. Phys.-Condens. Matter 10, 7121 (1998).

[30] M. L. Henle, and A. J. Levine, Phys. Rev. E 75, 059909 (2007).

[31] B. Pottier et al., Phys. Rev. E 89, 052403 (2014).

[32] A detailed computation is necessary to show the frequency dependence of the effect of Van der Waals forces on the fluctuations (from T. Bickel, private communication, and forthcoming paper). Evidence for the absence of such an effect is nevertheless provided by the master curve we obtain for $\omega^{2} S(\omega)$ as a function of $h$ and for small thickness, which is the same for all the probed frequencies.

[33] M. Thiebaud, and T. Bickel, Phys. Rev. E 81, 031602 (2010).

[34] M. Cieplak, J. Koplik, and J. R. Banavar, Phys. Rev. Lett. 86, 803 (2001).

[35] S. T. Cui, P. T. Cummings, and H. D. Cochran, J. Chem. Phys. 114, 6464 (2001). 